TI 2016-016/I

Tinbergen Institute Discussion Paper

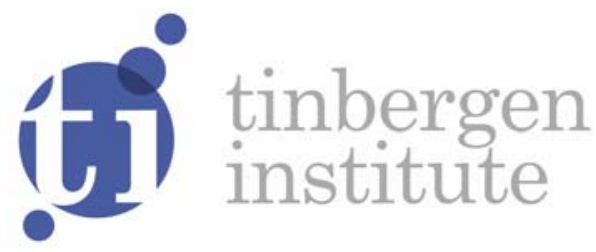

\title{
Sequential Auctions with Generalized Interdependent Values
}

\author{
Audrey Hu \\ Liang Zou
}

Faculty of Economics and Business, University of Amsterdam, and Tinbergen Institute, the Netherlands. 
Tinbergen Institute is the graduate school and research institute in economics of Erasmus University Rotterdam, the University of Amsterdam and VU University Amsterdam.

More TI discussion papers can be downloaded at http://www.tinbergen.nl

Tinbergen Institute has two locations:

Tinbergen Institute Amsterdam

Gustav Mahlerplein 117

1082 MS Amsterdam

The Netherlands

Tel.: +31(0)20525 1600

Tinbergen Institute Rotterdam

Burg. Oudlaan 50

3062 PA Rotterdam

The Netherlands

Tel.: +31(0)10 4088900

Fax: +31(0)10 4089031 


\title{
Sequential Auctions with Generalized Interdependent Values
}

\author{
Audrey $\mathrm{Hu}$ \\ University of Amsterdam/Tinbergen Institute, \\ 1018 TV Amsterdam, the Netherlands. \\ E-mail:X.Hu@uva.nl. \\ Liang Zou \\ University of Amsterdam, 1018 TV Amsterdam, the Netherlands. \\ E-mail:l.zou@uva.nl.
}

12 March, 2016 


\begin{abstract}
A common assumption in the analysis of symmetric auctions is that the bidders' value estimates exhibit positive informational externalities (PIE). This assumption implies upward drifting price sequences at sequential auctions, which is challenged by an empirical regularity, known as the "declining price anomaly," that observed price sequences at real sequential auctions tend to be downward-drifting. This paper extends the existing analysis to a generalized interdependent values environment, in which the bidders' values can exhibit both PIE and NIE (negative informational externalities). The case of NIE can arise naturally when competing bidders are also competitors in the same product market. If a bidder's type is related to his or his firm's ensuing competitive advantage, then an increase of a bidder's type increases his own but may decrease other bidders' expected values.

We consider a general sequential auction mechanism that sells $m$ identical objects through $K(\leq m)$ consecutive rounds, each round involving possibly a different number of objects for sale and a different payment rule. For risk neutral bidders having unit demand and independent types, we obtain two major results. First, the direct sequentially incentive compatible auction mechanisms, which implement the performance of essentially all standard auctions, are feasible under both PIE and NIE. Second, while the total expected revenue is invariant to sequencing and payment rules, the expected selling prices from different rounds of the auction are not the same. In a PIE environment the expected price sequence tends to be upward drifting, whereas in an NIE environment the expected price sequence is strongly downward drifting: the expected lowest price in round $k$ exceeds the expected highest price in round $k+1$. The declining price "anomaly" could, therefore, be evidence of bidders' values featuring NIE or post-auction competition.
\end{abstract}

Key words. Sequential auction, generalized interdependent values, declining price anomaly, informational externalities, revenue equivalence

JEL classification. D44, D82 


\section{Introduction}

One of the most interesting questions to ask about a sequential auction is: Does the equilibrium price sequence of selling multiple, identical objects entails any predictable trend? A good number of empirical studies have reported downward drifting patterns of the realized prices 11 This empirical regularity, known as the "declining price anomaly," poses a serious challenge to the standard auction theory which predicts, instead, flat (martingale) or increasing (submartingale) price trends at sequential auctions (e.g., Weber, 1983; Milgrom and Weber, 2000). While risk aversion provides a plausible explanation for declining prices, this conclusion has been derived only for the private values case (e.g., McAfee and Vincent, 1993; Mezzetti, 2011; Hu and Zou, 2015) ${ }^{2}$ Risk aversion does not necessarily imply declining prices under positive informational externalities (PIE) such that all bidders' values are nondecreasing functions of every other bidder's type or signal (e.g., Mezzetti, 2011).

In this paper, we present a sequential auction model with generalized interdependent values. The model allows for negative informational externalities (NIE) such that an increase of any bidder's type increases his own but decreases other bidders' values $3^{3}$ Casual observations suggest that competing bidders at a sequential auction are, typically, businesspersons or firms that are also competitors in the same product/service market. Apart from systematic factors or news that affect bidders' values

\footnotetext{
${ }^{1}$ For example, see Ashenfelter (1989), Ashenfelter and Genesove (1992); Beggs and Graddy (1997); McAfee and Vincent (1993); Milgrom and Weber (2000); Van den Berg, van Ours and Pradhan (2001); among others.

${ }^{2}$ There is also a voluminous literature seeking various institutional details that may rationalize the declining price phenomenon. E.g., Black and De Meza (1992); McAfee and Vincent (1993, 1997); Bernhardt and Scoones (1994); Engelbrecht-Wiggans (1994); Gale and Hausch (1994); Menezes and Monteiro (2003); Von der Fehr (1994); Jeitschko (1999); Gale and Stegeman (2001); Pitchick and Schotter (1988); Beggs and Graddy (1997); Ginsburgh (1998); Eyster (2002); Kittsteiner et al. (2004); and Rosato (2014), among others.

${ }^{3}$ The case with NIE has been considered in the more general asymmetric single-unit auction models, e.g., Krishna (2003) and Hu, Matthews, and Zou (2015).
} 
in common, a bidder's willingness-to-pay can also be related to the private information about his (firm's) perceived profitability upon winning an auctioned object. The information could, therefore, indicate a certain (anticipated) advantage in the ensuing product market competition 4 For instance, bidders at sequential flower auctions are mostly wholesalers or exporters..$^{5}$ When a bidder shows an exceptionally high willingness-to-pay for, say, roses on a certain day, it could be because he has received an unusually large buying order, or depleted his inventory sooner than usual, or acquired favorable information about his future market share. In any of these situations, it is not inconceivable that other bidders may interpret a competing bidder's strength as "bad news" and, as a result, reduce their own forecast profitability from buying the same roses. Of course, we view NIE as complementary to PIE, as both situations are possible.

Our model extends the existing literature also regarding the sequencing rules and payment rules of sequential auctions. Given $m(\geq 1)$ identical objects and $n(>m)$ bidders with unit demand, we allow the auctioneer to freely choose any sequencing rule, defined by the number of rounds $K(\leq m)$ and the number of objects $\ell_{k}$ to be auctioned in a round $k=1, \ldots, K$ such that $\sum_{k=1}^{K} \ell_{k}=m 6^{6}$ We also allow the auctioneer to freely choose any payment rule for each round of the auction so long as the rule is feasible. Thus, different rounds can involve different payment rules. We take a general approach of modelling sequential auctions as a sequence of direct mechanisms in which the strategy of every bidder is simply a sequence of reports of his

\footnotetext{
${ }^{4}$ See, e.g., Goeree (2003) for an analysis of auctions with aftermarket competitions, where bidders attempt to signal at the auction via the winning bid. We do not consider strategic signalling in this paper.

${ }^{5}$ See, e.g., van den Berg, van Ours, and Pradhan (2001) for an excellent empirical analysis of the Dutch flower auctions.

${ }^{6}$ This kind of general sequencing rules have been considered in Mezzetti, Pekeč and Tsetlin (2008) for the case of a two-round uniform sequential auction with affiliated types. Most of the sequential auction models consider selling a single unit of the objects in a round, and many restrict attention to the case with two rounds.
} 
type. A feasible sequential auction is one that has the property of sequential incentive compatibility (SIC) by which all active bidders report their types truthfully in every round. In order to obtain clear results, we focus on the case with risk neutral bidders and independent types.7

Under very mild conditions that the auctioned objects are valuable to all bidders, and that each bidder's valuation function increases in his own type, we show in Proposition 1 the existence and characterization of a general class of SIC auctions. A rather profound insight from this proposition is that essentially all standard auction policies can be implemented via a simple bidding rule that requires active bidders to report (or bid) their expected payment in each round. The formula that characterizes the SIC auctions allows us to provide an instructive proof of Corollary 1, which states that the total expected revenue is the same under any sequencing and payment rules. Corollaries $2-3$ illustrate how the characterization of the SIC auctions implies straightforwardly the characterizations of symmetric equilibria of the sequential allpay, discriminatory, and uniform auctions.

In Proposition 2, we investigate how information externalities affect the price trends in an SIC sequential auction. We find that, while the expected price sequences in the PIE environment tend to be upward drifting (extending, e.g., Milgrom and Weber, 2000; Mezzetti, 2011), in the NIE environment the price sequences are necessarily downward drifting. An interesting observation is that when multiple units of objects are sold in each round, the predictions of expected price trends are asymmetric. Under PIE, the expected highest price in round $k$ is predictably lower than that in round $k+1$; under NIE, the expected lowest price in round $k$ exceeds the expected highest price in round $k+1$. Thus, the downward price trends in the NIE environment

\footnotetext{
${ }^{7}$ The analysis presented in this paper can be further generalized to the case where bidders are risk averse, in a setting similar to $\mathrm{Hu}$ and Zou (2015). The analysis of sequential auctions with affiliated signals, however, remains a challenge (see Milgrom and Weber, 2000). While focusing on a two-stage uniform auction, Mezzetti, Pekeč and Tsetlin (2008) obtain the equilibrium existence and some important insights where bidders' signals are affiliated.
} 
are much more pronounced. These theoretical predictions suggest that the declining prices at real sequential auctions may be indeed associated with the fact that bidders at these auctions are competitors in the same product or service market.

The rest of the paper is organized as follows. In Section 2, we introduce the general environment with a formal definition of what we mean by PIE and NIE in this environment. We also describe the general sequential auction as a sequence of direct mechanisms, and characterize the conditions of sequential incentive compatibility. Section 3 presents the main results of the paper, including a numerical example in the end. Section 5 concludes the paper with remarks on possible extensions of the present study. Appendix A provides the proof of Proposition 1. Appendix B discusses the revenue equivalence result in Corollary 1 in more detail, including an instructive proof of the result that does not take the standard envelope theorem approach.

\section{Environment}

There are $m(\geq 1)$ identical objects for sale and $n(>m)$ competing bidders with unit demand. The reserve prices are zero. The sales are conducted using a general sequential auction involving a triplet of rules: a sequencing rule, an allocation rule, and a payment rule. The sequencing rule is defined by the number of rounds $K$ $(1 \leq K \leq m)$ and the number $\ell_{k}(\geq 1)$ of the objects to be simultaneously auctioned in round $k$ such that $\sum_{k=1}^{K} \ell_{k}=m$. In each round $k \in\{1, \ldots, K\}$, every active bidder $i$ submits a sealed "bid" $b_{i}^{k} \in \mathbb{R}_{+}$and the allocation rule is such that the highest $\ell_{k}$ bidders win. The winning bidders leave the auction upon paying according to the payment rule, while losing bidders remain active until all $m$ objects are sold.

The payment rule is defined, in general, by a function $p^{k}: \mathbb{R}_{+}^{n} \rightarrow \mathbb{R}_{+}$for $k=$ $1, \ldots, K$ as follows. At the start of round $k$, let $m_{k-1}=\sum_{j=1}^{k-1} \ell_{j}$ denote the total objects sold $\left(m_{0}:=0\right), \bar{b}_{m_{k-1}}$ the vector of previous winning bids, and $n_{k}=n-m_{k-1}$ the number of active bidders. When bidder $i$ bids $b_{i}^{k}$, his payment in round $k$ will be $p^{k}\left(b_{i}^{k} ; b_{-i}^{k}, \bar{b}_{m_{k-1}}\right)$, where $b_{-i}^{k}:=\left(b_{1}^{k}, \ldots b_{i-1}^{k}, b_{i+1}^{k}, \ldots, b_{n_{k}}^{k}\right)$. The function $p^{k}$ is symmetric 
in its last $n-1$ arguments, and satisfies $p^{k}\left(0 ; b_{-i}^{k}, \bar{b}_{m_{k-1}}\right) \equiv 0$. It is possible that both winners and losers pay (or receive) in each round.

Notice that $p^{k}\left(b_{i}^{k} ; b_{-i}^{k}, \bar{b}_{m_{k-1}}\right)$ can be also written as $p^{k}\left(b_{(i)}^{k} ; b_{-(i)}^{k}, \bar{b}_{m_{k-1}}\right)$, where $\left(b_{(i)}^{k}, b_{-(i)}^{k}\right)$ are obtained by rearranging $\left(b_{i}^{k}, b_{-i}^{k}\right)$ in decreasing order so that $b_{(1)}^{k} \geq$ $b_{(2)}^{k} \geq \ldots \geq b_{\left(n_{k}\right)}^{k}$. If several bidders are tied in any round $k$ with the same smallest winning bid $b_{\left(m_{k}\right)}^{k}$, then all of them will buy the remaining objects for the same price $p^{k}\left(b_{\left(m_{k}\right)}^{k} ; b_{-\left(m_{k}\right)}^{k}, \bar{b}_{m_{k-1}}\right)$. If the remaining number of objects falls short of the number of the winning bids in any round, then the allocation to the tied bidders will be resolved randomly, and the auction concludes.

The sequencing rule described above is completely general; as special cases, of course, are the most studied cases of the single-round simultaneous auction $(K=1)$ and the $m$-round sequential unit-sale auction $(K=m)$. The payment rule is also very general; apart from its flexibility for each round the functional form of $p^{k}$ can also differ from round to round. The following are three special cases of $p^{k}$ :

Sequential all-pay auction. All active bidders pay their own bids regardless of winning or losing in each round $k$ :

$$
p^{k}\left(b_{i}^{k}, b_{-i}^{k}, \bar{b}_{m_{k-1}}\right)=b_{i}^{k}
$$

Sequential uniform auction. All winners in round $k$ pay the "marketclearing" price: 8

$$
p^{k}\left(b_{i}^{k}, b_{-i}^{k}, \bar{b}_{m_{k-1}}\right)=\left\{\begin{array}{cl}
b_{\left(m_{k}+1\right)}^{k} & \text { if } b_{i}^{k} \geq b_{\left(m_{k}\right)}^{k} \\
0 & \text { if } b_{i}^{k}<b_{\left(m_{k}\right)}^{k}
\end{array}\right.
$$

Sequential discriminatory auction. All winners in round $k$ pay their own bids:

$$
p^{k}\left(b_{i}^{k}, b_{-i}^{k}, \bar{b}_{m_{k-1}}\right)=\left\{\begin{array}{cl}
b_{i}^{k} & \text { if } b_{i}^{k} \geq b_{\left(m_{k}\right)}^{k} . \\
0 & \text { if } b_{i}^{k}<b_{\left(m_{k}\right)}^{k}
\end{array}\right.
$$

\footnotetext{
${ }^{8}$ The market-clearing price is defined by the highest losing bid in this example. It can be defined more generally by any specific rule that determines a price between the highest losing bid and the lowest winning bid.
} 
Turning to bidders' preferences, we assume that every bidder $i \in N=\{1, \ldots, n\}$ has a private type $x_{i} \in[0,1]$. The type vector $\left(x_{1}, \ldots, x_{n}\right)$ is the realization of $n$ i.i.d. random variables $\left(X_{1}, \ldots, X_{n}\right) \in[0,1]^{n}$. We let $f$ and $F$ denote the density and cumulative distribution of $X_{i}$ and assume that $f$ is positive and continuous on $[0,1]$. Given $\left(x_{1}, \ldots, x_{n}\right)$, the (expected) value of the auctioned object to buyer $i$ is $v^{i}\left(x_{1}, \ldots, x_{n}\right)$. We assume there is a continuously differentiable function $v:[0,1]^{n} \rightarrow \mathbb{R}_{+}$ such that $v^{i}\left(x_{1}, \ldots, x_{n}\right) \equiv v\left(x_{i}, x_{-i}\right)$, and $v$ is invariant to permutations of its last $n-1$ arguments (e.g., Milgrom and Weber, 1982, 2000; Eso and White, 2004; Mezzetti, 2011). The function $v$ further satisfies the following condition $\sqrt[9]{ }$

A1. For all $\left(x_{1}, \ldots, x_{n}\right) \in[0,1]^{n}, 0 \leq v\left(x_{i}, x_{-i}\right)<\infty$ and $v_{1}\left(x_{i}, x_{-i}\right)>0$.

This mild and commonly made assumption is fairly basic. It says that the object is valuable to every bidder if he can acquire it free of charge, and that the bidder's value increases in his own type. We highlight two important cases that both satisfy A1 10

Case 1 The partial derivatives $v_{j}\left(x_{i}, x_{-i}\right) \geq 0$ for all $j=2, \ldots, n$.

Case 2 The partial derivatives $v_{j}\left(x_{i}, x_{-i}\right) \leq 0$ for all $j=2, \ldots, n$.

While Case 1 is the common assumption of PIE (e.g., Milgrom and Weber, 1982, 2000; Mezzetti, 2011), Case 2 involves NIE, which has been rarely studied in the symmetric auctions literature. One of the contributions of this paper is to show that the basic assumption A1 is, in fact, sufficient for the existence of pure strategy equilibria for a wide class of sequential auctions. This fact allows a researcher to analyze properties of sequential auction equilibria in environments accommodating Case 1, Case 2, and the full range of intermediate cases.

\footnotetext{
${ }^{9}$ Subscripts of $v$ denote partial derivatives and, as usual, these are defined as one-sided derivatives on the boundary of $[0,1]^{n}$.

${ }^{10}$ By symmetry, the two complementary conditions in Cases 1 and 2 can also be written as $v_{2} \geq 0$ and $v_{2} \leq 0$, respectively.
} 
We assume bidders are risk neutral, so that if bidder $i$ with type $x_{i}$ wins an object and pays price $p$, and if the type vector of other bidders is $x_{-i}$, the bidder has a payoff equal to $v\left(x_{i}, x_{-i}\right)-p$. Of course, the actual payoff is uncertain to the bidder as it depends on variables not observable by him at the time of the auction. A bidder's "status-quo" payoff, i.e., his utility level without participating in the auction, is assumed to be zero by normalization.

By symmetry, we focus w.l.o.g. on the decision of bidder 1 with type $x$. Define $\bar{Y}=\left(Y_{n-1}, \ldots, Y_{1}\right)$, where $Y_{k}$ is the $k$ th highest type from among the $n-1$ bidders other than bidder 1. Let $y_{j}$ denote a realization of $Y_{j}$. By symmetry of $v\left(x_{i}, x_{-i}\right)$ in $x_{-i}$, the valuation function of bidder 1 can also be written as $v(x, \bar{Y})$. To further ease notation, we denote $\bar{Y}_{m_{k}}=\left(Y_{m_{k}}, \ldots, Y_{1}\right)$ and $\bar{y}_{m_{k}}$ a realization of $\bar{Y}_{m_{k}}$.

\section{Sequential incentive compatibility}

By the revelation principle, we may perceive, as we do, a sequential auction as a sequence of direct mechanisms under which the bidders' bids are simply their "reported types." Conforming to certain real situations (e.g., a sequential Dutch auction), we assume that the mechanism (or auctioneer) announces the winning bids in each round $k$, and does not "record" the losing bidders' bids. ${ }^{11}$ We are interested in direct sequential auctions that are sequentially incentive compatible (SIC), under which every active bidder finds it optimal to report his type truthfully in any round of the auction - given that other bidders report their types truthfully (with probability 1 ) in all rounds, and that he plans to bid truthfully in subsequent rounds should he lose in the current round (see Definition 1 below). Therefore, SIC implies that all active bidders are informed of the vector of the previous winning types $\bar{y}_{m_{k-1}}$ at the start of every round $k$, and for bidder 1 with type $x$, he wins the $k$ th round if and only if $Y_{m_{k}} \leq x \leq Y_{m_{k-1}}$ $\left(Y_{m_{0}}:=1\right)$.

\footnotetext{
${ }^{11}$ The analysis of sequential auctions with affiliated values is more involved for the case without winning bids announcement. For example, see Mezzetti, Pekeč and Tsetlin (2008).
} 
A complete description of bidder 1's updated information should include the observation of 0 -probability reports. Suppose a winner in a round $k$ reported $z \geq$ $y_{m_{k-1}}$. This may happen, e.g., that the bidder, "by mistake," overstated his type in round $k$, or had understated his type in some previous rounds. In this case, we assume that all active bidders will assess this round- $k$ winner's type to be $y_{m_{k-1}}$. A complete description of bidder 1's strategies should also include the case where he himself has made a "mistake" by not bidding truthfully in some previous rounds. Then, truthful bidding in round $k$ could become suboptimal when the bidder's type $x$ exceeds $y_{m_{k-1}}$. In this case, knowing that the highest type from among other active bidders is less than $y_{m_{k-1}}$, the optimal strategy of bidder 1, as will be shown in the proof of Proposition 1, is to report $y_{m_{k-1}}$ and to win the $k$ th round with certainty.

To ease notation, define

$$
u^{k}\left(x, y, \bar{y}_{m_{k-1}}\right)=E\left[v(x, \bar{Y}) \mid \bar{Y}_{m_{k-1}}=\bar{y}_{m_{k-1}}, Y_{m_{k}}=y\right]
$$

as the $k$ th round conditional expected value of bidder 1 given that he is still active, that $\bar{Y}_{m_{k-1}}=\bar{y}_{m_{k-1}}$, and that his "closest" competitor in round $k$ has a type $Y_{m_{k}}$ equal to $y$. Given any payment rule $\left\{p^{k}: k=1, \ldots, K\right\}$, further define the active bidder 1 's expected payment in round $k$ by ${ }^{12}$

$$
\varphi^{k}\left(z, \bar{y}_{m_{k-1}}\right)=E\left[p^{k}(z, \bar{Y}) \mid \bar{Y}_{m_{k-1}}=\bar{y}_{m_{k-1}}\right]
$$

when he reports $z$. The bidder's $k$ th round conditional expected payoff when his type is $x$ and he reports $z$ thus equal: 13

$$
\begin{aligned}
V^{k}\left(z, x \mid \bar{y}_{m_{k-1}}\right): & =E\left[u^{k}\left(x, Y_{m_{k}}, \bar{y}_{m_{k-1}}\right) \mid\left\{Y_{m_{k}} \leq z\right\}\right]-\varphi^{k}\left(z, \bar{y}_{m_{k-1}}\right) \\
& +E\left[V^{k+1}\left(x \wedge Y_{m_{k}}, x \mid \bar{Y}_{m_{k}}\right) \mid\left\{Y_{m_{k}}>z\right\}, \bar{Y}_{m_{k-1}}=\bar{y}_{m_{k-1}}\right]
\end{aligned}
$$

\footnotetext{
${ }^{12}$ In light of Proposition 1, we suppress the notational dependence of $\varphi^{k}$ on function $p^{k}$.

${ }^{13}$ The term $E\left(\cdot \mid\left\{Y_{m_{k}} \leq z\right\}\right)$ denotes the unconditional expectation under the "event" $\left\{Y_{m_{k}} \leq z\right\}$ so that $E\left(\cdot \mid\left\{Y_{m_{k}} \leq z\right\}\right)=E\left(\cdot \mid Y_{m_{k}} \leq z\right\} \operatorname{Pr}\left(\left\{Y_{m_{k}} \leq z\right\}\right)$. Note that this expectation is invariant to the tie-breaking rules since $Y_{m_{k}}=z$ occurs with probability of zero.
} 
where $V^{K+1}:=0$ and $x \wedge y=\min \{x, y\}$. In (6), the first term on the right-hand side is associated with the event of bidder 1 winning the $k$ th round, the second term his expected payment, and the last term the event of him losing the $k$ th round. If the bidder loses in any round $k<K$, his subsequent conditional expected payoff given an updated $\bar{y}_{m_{k}}$ is $V^{k+1}\left(x \wedge Y_{m_{k}}, x \mid \bar{y}_{m_{k}}\right)$. The term $x \wedge Y_{m_{k}}$ captures the possibility that the bidder reported a type lower than $x$, losing the $k$ th round, and learned that $x>y_{m_{k}}$. In this case his optimal subsequent strategy is to report $y_{m_{k}}$ instead of $x$ in the $(k+1)$ st round, as is shown in the proof of Proposition 1 .

Definition $1 A$ direct sequential auction with sequencing rule $\left\{\ell_{k}, k=1, \ldots, K\right\}$, payment rule $\left\{p^{k}, k=1, \ldots, K\right\}$, and allocation rule that the highest $\ell_{k}$ bidders win in each round $k=1, \ldots, K$ is sequentially incentive compatible (SIC) iff

$$
\begin{aligned}
V^{k}\left(x, x \mid \bar{y}_{m_{k-1}}\right) & \geq V^{k}\left(z, x \mid \bar{y}_{m_{k-1}}\right) \\
\forall \bar{y}_{m_{k-1}} & \in[0,1]^{m_{k-1}}, \forall z, x \in[0,1], \forall k \in\{1, \ldots, K\} .
\end{aligned}
$$

We say that an SIC sequential auction is feasible if $V^{k}\left(x, x \mid \bar{y}_{m_{k-1}}\right) \geq 0$ for all $x$ and $\bar{y}_{m_{k-1}}, k=1, \ldots, K$. Clearly, by A1 and $p^{k}(0 ; \cdot) \equiv 0$, all SIC sequential auctions are feasible.

An important aspect of our formulation of sequential incentive compatibility is that bidders are "forward looking" in making their bidding decisions. A losing bidder up to round $k$ may have well suffered certain losses already, (e.g., when the payment rule resembles an all-pay auction), or have made certain gains (e.g., when the payment rule resembles a "Santa Clause" auction as described in Riley and Samuelson (1981)). We assume that these "sunk costs" or "locked-in gains" do not affect the bidders' current and future decisions.

\section{Main Results}

We are now ready to investigate the major issues regarding equilibrium existence, price trends, and expected revenue. Let $F_{m_{k}}\left(\cdot \mid \bar{y}_{m_{k-1}}\right)$ denote the cumulative distribution, 
and $f_{m_{k}}\left(\cdot \mid \bar{y}_{m_{k-1}}\right)$ the associated density function, of $Y_{m_{k}}$ conditional on $\bar{Y}_{m_{k-1}}=\bar{y}_{m_{k-1}}$. Recall that $m_{k-1}+\ell_{k}=m_{k}$ and $n_{k}=n-m_{k-1}$, where $\ell_{k}$ is the number of objects to be sold in round $k$. Thus,

$$
f_{m_{k}}\left(y \mid \bar{y}_{m_{k-1}}\right)=\frac{\left(n_{k}-1\right) !}{\left(n_{k}-\ell_{k}-1\right) !\left(\ell_{k}-1\right) !} \frac{F(y)^{n_{k}-\ell_{k}-1}\left(F\left(y_{m_{k-1}}\right)-F(y)\right)^{\ell_{k}-1} f(y)}{F\left(y_{m_{k-1}}\right)^{n_{k}-1}}
$$

As a consequence of $X_{i}^{\prime}$ 's being i.i.d., the distribution $F_{m_{k}}\left(\cdot \mid \bar{y}_{m_{k-1}}\right)$ depends only on $y_{m_{k-1}}$ so that we will write it as $F_{m_{k}}\left(\cdot \mid y_{m_{k-1}}\right)$.

Our first proposition establishes the existence and characterization of the general class of feasible sequential auctions.

Proposition 1 Suppose A1 holds. Then, under any sequencing rule $\left\{\ell_{k}: k=\right.$ $1, \ldots, K\}$ and allocation rule such that the highest $\ell_{k}$ bidders win in each round $k$, there exist feasible direct sequential auctions. The payment rule $\left\{p^{k}, k=1, \ldots, K\right\}$ of all feasible sequential auctions imply the same expected payment function $\varphi^{k}\left(\cdot, \bar{y}_{m_{k-1}}\right)$ characterized by

$$
\varphi^{K}\left(x, \bar{y}_{m_{K-1}}\right)=\int_{0}^{x} u^{K}\left(y, y, \bar{y}_{m_{K-1}}\right) d F_{m}\left(y \mid y_{m_{K-1}}\right),
$$

and for $k=1, \ldots, K-1, \sqrt[14]{14}$

$$
\varphi^{k}\left(x, \bar{y}_{m_{k-1}}\right)=\int_{0}^{x} E\left[\varphi^{k+1}\left(y, y, Y_{m_{k}-1}, \ldots, Y_{m_{k-1}+1}, \bar{y}_{m_{k-1}}\right)\right] d F_{m_{k}}\left(y \mid y_{m_{k-1}}\right)
$$

Moreover, all $\varphi^{k}\left(x, \bar{y}_{m_{k-1}}\right)$ are positive and increasing in $x$ on $\left(0, y_{m_{k-1}}\right)$.

Proof. See Appendix A.

The result of this proposition is remarkably general, as it requires only the basic assumption A1. In the characterization of the final-round bidding strategy, equation (8) resembles that of a single-round simultaneous auction except that the active bidders have now the updated information of the previous winning bidders' types. Indeed, it reduces to the characterization of incentive compatible direct mechanisms

\footnotetext{
${ }^{14}$ Note that for $\ell_{k}=2$, the vector $\left(Y_{m_{k}-1}, \ldots, Y_{m_{k-1}+1}\right)=Y_{m_{k}-1}=Y_{m_{k-1}+1}$. And by convention, for $\ell_{k}=1$, we interpret $\left(Y_{m_{k}-1}, \ldots, Y_{m_{k-1}+1}\right)$ as nonexistent.
} 
for the simultaneous multi-unit auction when we set $K=1$ (with $y_{m_{0}}=1$ ). For the more general case, the equations in (9) show that in each round $k$, an active bidder equates his expected payment with the expected payment by his closest competitor, the one with type $Y_{m_{k}}$, in the subsequent round assuming that the competitor would be tied with another bidder with the same type $Y_{m_{k}}$.

By (8) and (9), an explicit expression of the expected round- $k$ payment can be deduced by working backward from the last period. For $k=1, \ldots, K-1$, we have

$$
\begin{aligned}
& \varphi^{k}\left(x, \bar{y}_{m_{k-1}}\right) \\
= & \int_{0}^{x} E\left[\varphi^{k+1}\left(y, y, Y_{m_{k}-1}, \ldots, Y_{m_{k-1}+1}, \bar{y}_{m_{k-1}}\right)\right] d F_{m_{k}}\left(y \mid y_{m_{k-1}}\right) \\
= & \int_{0}^{x} E\left[\varphi^{k+1}\left(Y_{m_{k}}, Y_{m_{k}}, Y_{m_{k}-1}, \ldots, Y_{m_{k-1}+1}, \bar{y}_{m_{k-1}}\right) \mid Y_{m_{k}}=y\right] d F_{m_{k}}\left(y \mid y_{m_{k-1}}\right) \\
& \quad \ldots \\
= & \int_{0}^{x} E\left[u^{K}\left(Y_{m}, Y_{m}, Y_{m_{K-1}}, \ldots, Y_{m_{k-1}+1}, \bar{y}_{m_{k-1}}\right) \mid Y_{m_{k}}=y\right] d F_{m_{k}}\left(y \mid y_{m_{k-1}}\right) \\
= & \int_{0}^{x} E\left[v\left(Y_{m}, \bar{Y}\right) \mid Y_{m_{k}}=y, \bar{Y}_{m_{k-1}}=\bar{y}_{m_{k-1}}\right] d F_{m_{k}}\left(y \mid y_{m_{k-1}}\right)
\end{aligned}
$$

where the last equation is due to (4). The following corollary is a direct consequence of Proposition 1 .

Corollary 1 (revenue equivalence) Under A1, (i) given any sequencing rule and any SIC payment rule, the expected revenue from round $k$ is the same; and (ii) any sequencing rule of a sequential auction implies the same total expected revenue ex-ante.

Proof. (i) This is an obvious consequence of (10), since all payment rules imply the same expected payment from a given round.

(ii) This follows from $\varphi^{k}\left(x, \bar{y}_{m_{k-1}}\right)$ being increasing in $x$, which implies that prior to the auction, all bidders have the same probability of winning an object under any sequencing and payment rules of an SIC auction. Since the bidder having type zero pays and receives nothing, by the revenue equivalence theorem every bidder has the same expected payoff prior to the auction. Hence the seller's total expected revenue 
must be invariant to sequencing and payment rules. We provide an instructive proof in Appendix B.

The general characterization of the SIC auctions in Proposition 1 provides a simple way to derive equilibria of the more specific auction policies. A rather profound insight from Proposition 1 is that under risk neutrality, there is no loss of generality to restrict attention to bidding rules that require bidders to submit bids equal to what they expect to pay in each round of a sequential auction so long as they are still active. The winner's actual payment can be then determined according to the pre-specified payment rules. The sequential all-pay auction as described in (1) is an immediate corollary of this result.

Corollary 2 (sequential all-pay auction) Suppose A1 holds in the environment described in Section 2, and the payment rules are "all pay" or "pay-your-bid" regardless of winning or losing in every round as in (1). Then, given any sequencing rule $\left\{\ell_{k}: k=1, \ldots, K\right\}$, there exists a continuous and increasing pure strategy symmetric equilibrium $\left\{b_{A P}^{k}: k=1, \ldots, K\right\}$ of the sequential all-pay auction. In each round $k$ given previous winners' type vector $\bar{y}_{m_{k-1}}$, the equilibrium strategy of an active bidder with type $x$ is to bid

$$
b_{A P}^{k}\left(x, \bar{y}_{m_{k-1}}\right)=\int_{0}^{x} E\left[v\left(Y_{m}, \bar{Y}\right) \mid Y_{m_{k}}=y, \bar{Y}_{m_{k-1}}=\bar{y}_{m_{k-1}}\right] d F_{m_{k}}\left(y \mid y_{m_{k-1}}\right)
$$

Proof. Simply define the bid function $b_{A P}^{k}$ as $\varphi^{k}$ defined in (8)-(9), or in (10), of Proposition 1 .

The result of this corollary is new. It is perhaps surprising that the equilibrium existence for the sequential all-pay auction, in the present model with generalized interdependent values and sequencing rules, requires only the basic assumption A1 that bidders like the items (e.g., a price, research grant, etc.), and that every bidder's value increases in his own type.

As for the sequential discriminatory and uniform auctions, we invoke an additional assumption to ensure monotonicity of the bid functions in the NIE environment (Case 2). 
A2. $E\left[v\left(Y_{m}, \bar{Y}\right) \mid Y_{j}=x, Y_{j-1}=y_{j-1}, \ldots, Y_{1}=y_{1}\right]$ is an increasing function of $x$, for all $j \leq m$ and $\left(y_{j-1}, \ldots, y_{1}\right)$.

This assumption holds naturally in the PIE environment of Case 1 when $v$ is nondecreasing in all its arguments and increasing in its first argument (see, e.g., Milgrom and Weber, 1982, Theorem 5). In the NIE environment of Case 2, A2 restricts the extent to which the NIE affects bidders' values. Roughly, A2 requires that increasing a bidder's own type has a dominant effect compared to increasing any competitor's type (in the sense of the stated condition). The following are some simple examples in which A2 holds under NIE.

Examples. There is no loss of generality to assume, as we do here, that every bidder's type $X_{i}$ is uniformly distributed on [0,1] (see, e.g., Milgrom, 2004, pp. 111). On the basis of this assumption, it can be verified that the following value functions satisfy A1-A2 for $\alpha<1 / n$ for arbitrary $m<n$ (tighter conditions can be computed as well):

$$
\begin{aligned}
\text { (i) } v\left(x_{1}, \ldots, x_{n}\right) & =x_{1}\left(1-\alpha\left(x_{2}+\ldots+x_{n}\right)\right) \\
\text { (ii) } v\left(x_{1}, \ldots, x_{n}\right) & =1+x_{1}-\alpha\left(x_{2}+\ldots+x_{n}\right) \\
\text { (iii) } v\left(x_{1}, \ldots, x_{n}\right) & =1+x_{1}-\alpha\left(x_{2} \times \ldots \times x_{n}\right)
\end{aligned}
$$

We are ready to present two more corollaries of Proposition 1 .

Corollary 3 (sequential uniform auction) Suppose in the environment described in Section 2, the payment rules are uniform in each round $k$ as defined in (2). Then, under A1-A2, given any sequencing rule $\left\{\ell_{k}: k=1, \ldots, K\right\}$, there exists a continuous and increasing pure strategy symmetric equilibrium $\left\{b_{U}^{k}: k=1, \ldots, K\right\}$ of the sequential uniform auction such that

$$
b_{U}^{k}\left(x, \bar{y}_{m_{k-1}}\right)=E\left[v\left(Y_{m}, \bar{Y}\right) \mid Y_{m_{k}}=x, \bar{Y}_{m_{k-1}}=\bar{y}_{m_{k-1}}\right]
$$

Proof. By the uniform payment rule defined in (2),

$$
\varphi^{k}\left(x, \bar{y}_{m_{k-1}}\right)=\int_{0}^{x} b_{U}^{k}\left(y, \bar{y}_{m_{k-1}}\right) d F_{m_{k}}\left(y \mid y_{m_{k-1}}\right)
$$


Substituting (15) into (10), and differentiating w.r.t. $x$ and cancelling terms, yields (14). A2 implies that $b_{U}^{k}\left(x, \bar{y}_{m_{k-1}}\right)$ is an increasing function of $x$. Hence the conclusion.

Corollary 4 (sequential discriminatory auction) Suppose in the environment described in Section 2, the payment rules are discriminatory in each round $k$ as defined in (3). Then, under A1-A2, given any sequencing rule $\left\{\ell_{k}: k=1, \ldots, K\right\}$, there exists a continuous and increasing pure strategy symmetric equilibrium $\left\{b_{D}^{k}: k=1, \ldots, K\right\}$ of the sequential discriminatory auction such that

$$
\begin{aligned}
& b_{D}^{k}\left(x, \bar{y}_{m_{k-1}}\right) \\
= & \frac{1}{F_{m_{k}}\left(x \mid y_{m_{k-1}}\right)} \int_{0}^{x} E\left[v\left(Y_{m}, \bar{Y}\right) \mid Y_{m_{k}}=y, \bar{Y}_{m_{k-1}}=\bar{y}_{m_{k-1}}\right] d F_{m_{k}}\left(y \mid y_{m_{k-1}}\right) \\
= & E\left[v\left(Y_{m}, \bar{Y}\right) \mid Y_{m_{k}} \leq x \leq y_{m_{k-1}}, \bar{Y}_{m_{k-1}}=\bar{y}_{m_{k-1}}\right]
\end{aligned}
$$

Proof. By (3), the expected payment of an active bidder 1 with type $x$ equals $\varphi^{k}\left(x, \bar{y}_{m_{k-1}}\right)=b_{D}^{k}\left(x, \bar{y}_{m_{k-1}}\right) F_{m_{k}}\left(x \mid y_{m_{k-1}}\right)$ in round $k$. Thus, 10) implies (16). Since A2 implies that the integrand in (16) increases in $y$, and since increasing $x$ shifts the conditional distribution $F_{m_{k}}\left(y \mid y_{m_{k-1}}\right) / F_{m_{k}}\left(x \mid y_{m_{k-1}}\right)$ of $y$ to the right, $b_{D}^{k}\left(x, \bar{y}_{m_{k-1}}\right)$ is an increasing function of $x$. Hence the conclusion.

Our next proposition provides clear predictions about the expected price trends over rounds of a sequential auction. We focus on general winners-pay sequential auctions. Other auction formats that involve losing bidders paying or receiving money can be investigated in terms of total proceeds, but these are of less empirical interest given the revenue equivalence result in Corollary 1 .

Proposition 2 Suppose A1 holds. Let any sequencing rule $\left\{\ell_{k}: k=1, \ldots, K\right\}$ be given and suppose that the payment rule is such that the losing bidders in every round pay and receive nothing. Denote by $E\left(\tilde{p}_{\min }^{k} \mid \bar{y}_{m_{k-1}}\right)$ and $E\left(\tilde{p}_{\max }^{k} \mid \bar{y}_{m_{k-1}}\right)$ the lowest and highest expected payment in round $k$, and by $E\left(\tilde{p}_{\max }^{k+1} \mid \bar{y}_{m_{k-1}}\right)$ the highest expected payment in round $k+1$ (under the same condition $\bar{Y}_{m_{k-1}}=\bar{y}_{m_{k-1}}$ ). Then, for all $k=1, \ldots, K-1$, 
(i) $E\left(\tilde{p}_{\max }^{k} \mid \bar{y}_{m_{k-1}}\right) \leq E\left(\tilde{p}_{\max }^{k+1} \mid \bar{y}_{m_{k-1}}\right)$ if Case 1 holds;

(ii) $E\left(\tilde{p}_{\min }^{k} \mid \bar{y}_{m_{k-1}}\right) \geq E\left(\tilde{p}_{\max }^{k+1} \mid \bar{y}_{m_{k-1}}\right)$ if Case 2 holds.

Proof. By (9) and (10), bidder 1 with type $x$ has an expected payment in round $k$ that equals

$$
\begin{aligned}
\varphi^{k}\left(x, \bar{y}_{m_{k-1}}\right) & =E\left[\varphi^{k+1}\left(Y_{m_{k}}, Y_{m_{k}}, Y_{m_{k}-1}, \ldots, Y_{m_{k-1}+1}, \bar{y}_{m_{k-1}}\right) \mid\left\{Y_{m_{k}} \leq x<y_{m_{k-1}}(\hat{j}] 7\right)\right. \\
& =E\left[v\left(Y_{m}, \bar{Y}\right) \mid \bar{Y}_{m_{k-1}}=\bar{y}_{m_{k-1}},\left\{Y_{m_{k}} \leq x<y_{m_{k-1}}\right\}\right]
\end{aligned}
$$

It can be readily argued, e.g., by Theorem 5 in Milgrom and Weber (1982), that the term in 18 is nondecreasing (nonincreasing) in every component of $\bar{y}_{m_{k-1}}$ in Case 1 (Case 2); that is,

$$
\frac{\partial}{\partial y_{j}} \varphi^{k}\left(x, \bar{y}_{m_{k-1}}\right)\left\{\begin{array}{l}
\geq 0 \text { in Case 1 } \\
\leq 0 \text { in Case } 2
\end{array}, \forall k \leq K, \forall j=1, \ldots, m_{k-1}\right.
$$

Consider part (ii) first, assuming Case 2 holds. The "event" that bidder 1 wins the $k$ th round corresponds to the event $\left\{Y_{m_{k}}<x<Y_{m_{k-1}}\right\}$. (We ignore the zero-probability events of $x=Y_{m_{k}}$ and $x=Y_{m_{k-1}}$.) In round $k+1$ and under event $\left\{Y_{m_{k}}<x<Y_{m_{k-1}}\right\}$, the bidder with type $Y_{m_{k}}$ will be the winner with the highest expected payment in that round, which, conditional on the realization of $\left(Y_{m_{k}-1}, \ldots, x, \ldots, Y_{m_{k-1}+1}, \bar{y}_{m_{k-1}}\right)$, equals

$$
\begin{aligned}
& E\left(\tilde{p}_{\max }^{k+1} \mid \bar{y}_{m_{k-1}},\left\{Y_{m_{k}}<x<Y_{m_{k-1}}\right\}\right) \\
= & E\left[\varphi^{k+1}\left(Y_{m_{k}}, x, Y_{m_{k}-1}, \ldots, Y_{m_{k-1}+1}, \bar{y}_{m_{k-1}}\right) \mid\left\{Y_{m_{k}}<x<Y_{m_{k-1}}\right\}\right] \\
\leq & E\left[\varphi^{k+1}\left(Y_{m_{k}}, Y_{m_{k}}, Y_{m_{k}-1}, \ldots, Y_{m_{k-1}+1}, \bar{y}_{m_{k-1}}\right) \mid\left\{Y_{m_{k}}<x<Y_{m_{k-1}}\right\}\right] \\
= & \varphi^{k}\left(x, \bar{y}_{m_{k-1}}\right) \text { for all } x \text { such that } Y_{m_{k}}<x<Y_{m_{k-1}},
\end{aligned}
$$

where 200 uses the fact that $\varphi^{k}(x, \cdot)$ is symmetric, 21) follows from 19), and 22 is (18). Since $\varphi^{k}\left(x, \bar{y}_{m_{k-1}}\right)$ increases in $x$, in the event that bidder 1 has $x=Y_{m_{k}-1}$, $\varphi^{k}\left(x, \bar{y}_{m_{k-1}}\right)=E\left(\tilde{p}_{\min }^{k} \mid \bar{y}_{m_{k-1}}\right)$. Hence the conclusion of (ii). 
Now consider part (i) of Case 1. Using similar arguments the inequality in 21) is reversed, so that

$$
E\left(\tilde{p}_{\max }^{k+1} \mid \bar{y}_{m_{k-1}},\left\{Y_{m_{k}}<x<Y_{m_{k-1}}\right\}\right) \geq \varphi^{k}\left(x, \bar{y}_{m_{k-1}}\right)
$$

for all $x$ such that $Y_{m_{k}}<x<Y_{m_{k-1}}$. In the event that bidder 1 has $x=Y_{m_{k-1}+1}$, his expected payment $\varphi^{k}\left(x, \bar{y}_{m_{k-1}}\right)=E\left(\tilde{p}_{\max }^{k} \mid \bar{y}_{m_{k-1}}\right)$. Hence the conclusion of (i).

It is interesting to observe that this proposition provides asymmetric predictions of expected price trends. For Case 1, the maximum expected payments can be compared over rounds, whereas for Case 2 the prediction of downward sloping price trend is much more pronounced: the minimum expected payment in round $k$ exceeds the maximum expected payment in round $k+1$. The reason for this asymmetry is due to equation (9), where the $k$ th round expected payment by all types of active bidders are related to the highest expected payment in the $(k+1)$ st round. Of course, for the special sequencing rule of $K=m$ where each round sells exactly one unit, we have the symmetric prediction that the expected price trend increases (decreases) under PIE (NIE).

An implication of Proposition 2 is that when there are multiple sellers, unless they agree to share the total proceeds from the sales, their expected payoffs can be sensitive to the rounds in which their items are allotted to, as well as to the nature of interdependence of bidders' values.

A Numerical Example. Consider the case of $(11)$, where $F(x)=x$ on $[0,1]$ and $v\left(x_{1}, \ldots, x_{n}\right)=x_{1}\left(1-\alpha\left(x_{2}+\ldots+x_{n}\right)\right)$ with $a<1 / 8$. Suppose $n=8, m=4$, and the seller chooses to sell one unit in a round, so that $K=4$. Suppose the seller chooses the Dutch auction format (which corresponds to a first-price sealed-bid auction with the winning bid announcement).

Let $b_{D}^{k}$ denote the equilibrium bid function in round $k$. By 10 , these are given by 


$$
\begin{aligned}
b_{D}^{1}(x ; a) & =-\frac{1}{36} x(67 a x-18) \\
b_{D}^{2}\left(x, y_{1} ; a\right) & =-\frac{1}{28} x\left(16 a y_{1}+51 a x-16\right) \\
b_{D}^{3}\left(x, y_{2}, y_{1} ; a\right) & =-\frac{1}{21} x\left(14 a y_{1}+14 a y_{2}+37 a x-14\right) \\
b_{D}^{4}\left(x, y_{3}, y_{2}, y_{1} ; a\right) & =-\frac{1}{15} x\left(12 a y_{1}+12 a y_{2}+12 a y_{3}+25 a x-12\right)
\end{aligned}
$$

It is easy to verify that for $a<1 / 8$, all $b_{D}^{k}(x, \cdot)$ are increasing functions of $x$. Note also that $b_{D}^{k}$ are nonincreasing functions of $y_{1}, y_{2}, y_{3}$ if and only if $a \geq 0$. From the seller's viewpoint, the expected revenue from different rounds are given by

\begin{tabular}{lllll}
$\mathrm{E}\left(b_{D}^{1}\right)$ & $\mathrm{E}\left(b_{D}^{2}\right)$ & $\mathrm{E}\left(b_{D}^{3}\right)$ & $\mathrm{E}\left(b_{D}^{4}\right)$ & Total \\
\hline \hline$\frac{4}{9}-\frac{67}{45} a$ & $\frac{4}{9}-\frac{23}{15} a$ & $\frac{4}{9}-\frac{71}{45} a$ & $\frac{4}{9}-\frac{73}{45} a$ & $\frac{16}{9}-\frac{56}{9} a$ \\
\hline
\end{tabular}

The table shows that the expected price trend increases for $a<0$ (PIE) and decreases for $a>0$ (NIE). If the seller conducts the sale using a simultaneous discriminatory auction, the total expected revenue can be computed as 16/9 - 56a/9, confirming revenue equivalence.

\section{Concluding Remarks}

This paper extends the existing analysis of sequential auctions in three aspects. First, bidders can exhibit generalized interdependent values including as polar cases positive informational externalities (PIE) and negative informational externalities (NIE). Second, the sequencing rule to sell $m$ identical objects is completely general: the seller/auctioneer has the discretion to decide the number of rounds $K(\leq m)$ and the number of objects to be sold in each round $k=1, \ldots, K$. And third, the payment rule for each round $k$ is arbitrary, which can differ from round to round so long as the bidder with type zero pays and receives nothing.

We take the general approach of analyzing sequential auctions as a sequence of direct mechanisms (i.e., the Myersonian approach; see Pavan, Segal, and Toikka, 
2014). We formulate the sequentially incentive compatible (SIC) constraints that necessarily limit the seller's choice of sequencing and payment rules, and show that all SIC sequential auctions that allocate the objects to the highest bidders have the same characterization regarding an active bidder's expected payment in each round. The formula characterizing the general SIC auctions lends itself to the derivation of pure strategy equilibria of the more standard sequential auctions. We show how the formula implies straightforwardly the equilibrium existence of sequential all-pay auctions and, under appropriate additional conditions, equilibrium existence of sequential discriminatory and uniform auctions. We use the general formula to show that the expected payoff/revenue is invariant to different payment rules in a given round and, less obviously, that the total expected payoff/revenue is invariant also to different sequencing rules as well.

In answering our motivating question concerning the price trends at a sequential auction, we obtain a surprisingly strong declining price result. We show that, in an NIE environment, all SIC sequential auctions imply that the expected lowest price in round $k$ exceeds the expected highest price in round $k+1$ given any sequencing rule. Thus, the "declining price anomaly" is not an anomaly; rather, it is a natural consequence of a bidding environment in which increasing a bidder's type increases the bidder's own value but decreases other bidders' values. As to the environment with PIE, we confirm the existing theoretical prediction that the price trends at sequential auctions should be upward drifting in that the maximum expected price in round $k$ is lower than the maximum expected price in round $k+1$. Since the PIE and NIE environments can be created easily in a laboratory, the predictions of this paper are highly amenable to experimental testing.

In order to obtain clear results, we have limited attention to situations where bidders are risk neutral, having unit demand and independent types. The analysis can be extended to situations where bidders are risk averse under conditions in $\mathrm{Hu}$ and Zou (2015). Risk aversion will further strengthen the downward trend of prices at sequential auctions under NIE, while blurring the price patterns under PIE (Mezzetti, 
2011). Extensions to multi-unit demand or affiliated types in the present setting with generalized interdependent values remain important open problems.

\section{Appendix A. Proof of Proposition 1}

Proof. Rewrite the expected payoff in (6) as

$$
\begin{aligned}
& V^{k}\left(z, x \mid \bar{y}_{m_{k-1}}\right)=\int_{0}^{z} u^{k}\left(x, y, \bar{y}_{m_{k-1}}\right) d F_{m_{k}}\left(y \mid \bar{y}_{m_{k-1}}\right)-\varphi^{k}\left(z, \bar{y}_{m_{k-1}}\right) \\
& +\int_{z}^{y_{m_{k-1}}} E\left[V^{k+1}\left(x \wedge y, x \mid \bar{Y}_{m_{k}}\right) \mid Y_{m_{k}}=y, \bar{Y}_{m_{k-1}}=\bar{y}_{m_{k-1}}\right] d F_{m_{k}}\left(y \mid \bar{y}_{m_{k-1}}\right)
\end{aligned}
$$

where $F_{m_{k}}\left(\cdot \mid \bar{y}_{m_{k-1}}\right)$ is given in (7). We analyze by backward induction bidder 1's optimal response, assuming that all others report truthfully their types in all rounds.

Round $K$. Suppose bidder 1 with type $x$ is active in the final round $K$, with updated information that $\bar{Y}_{m_{K-1}}=\bar{y}_{m_{K-1}}$. By $(23)$, reporting a type $z$ leads to an expected payoff

$$
V^{K}\left(z, x \mid \bar{y}_{m_{K-1}}\right)=\int_{0}^{z} u^{K}\left(x, y, \bar{y}_{m_{K-1}}\right) d F_{m}\left(y \mid \bar{y}_{m_{K-1}}\right)-\varphi^{K}\left(z, \bar{y}_{m_{K-1}}\right)
$$

Differentiating w.r.t. $z$ gives

$$
V_{1}^{K}\left(z, x \mid \bar{y}_{m_{K-1}}\right)=u^{K}\left(x, z, \bar{y}_{m_{K-1}}\right) f_{m}\left(z \mid \bar{y}_{m_{K-1}}\right)-\frac{\partial}{\partial z} \varphi^{K}\left(z, \bar{y}_{m_{K-1}}\right)
$$

Since $v_{1}>0, u^{K}\left(x, z, \bar{y}_{m_{K-1}}\right)$ increases in $x$ and so does $V_{1}^{K}\left(z, x \mid \bar{y}_{m_{K-1}}\right)$. Therefore, $V^{K}\left(z, x \mid \bar{y}_{m_{K-1}}\right)$ satisfies the single-crossing condition of Milgrom and Shannon (1994) (see also Athey, 2001). This implies that $V_{1}^{K}\left(x, x \mid y_{m-1}\right)=0$ is both necessary and sufficient for SIC to hold in round $K$ provided $x \leq y_{m_{k-1}}$. By (24), integrating $V_{1}^{K}\left(y, y \mid \bar{y}_{m_{K-1}}\right)=0$ over $y \in[0, x]$ gives

$$
\varphi^{K}\left(x, \bar{y}_{m_{K-1}}\right)=\int_{0}^{x} u^{K}\left(y, y, \bar{y}_{m_{K-1}}\right) d F_{m}\left(y \mid \bar{y}_{m_{K-1}}\right)
$$

The existence and uniqueness of $\varphi^{K}\left(x, \bar{y}_{m_{K-1}}\right)$ follows from the fact that the right-hand side of 25$)$ is a well defined function of $\left(x, \bar{y}_{m_{K-1}}\right)$. This function is positive and increasing in $x$ because $u^{K}\left(y, y, \bar{y}_{m_{K-1}}\right)$ is positive by A1. 
As we do not assume that bidder 1 has followed the equilibrium strategies previously, there is also a possibility that $x>y_{m_{K-1}}$. This case is only possible if in the preceding round the bidder has deviated from the equilibrium strategy and bid as though his type were lower than $x$, for else he would have won the previous round. Given $y_{m} \leq y_{m_{K-1}}<x$, it does not make sense to bid above $y_{m_{K-1}}$; it is optimal for the bidder to bid $z=y_{m_{K-1}}<x$ and win the $K$ th round with probability 1 . Thus, the complete bidding strategy in the final round is to bid $x \wedge y_{m_{K-1}}\left(=\min \left\{x, y_{m_{K-1}}\right\}\right)$. This, of course, will not be the case when the bidder has followed the equilibrium strategy from the start.

Round $k$. Now consider $k<K$. Our induction hypothesis is that given any $\bar{y}_{m_{k}}$, the expected payment function $\varphi^{k+1}\left(x, \bar{y}_{m_{K}}\right)$ is well defined, positive for $x>0$, increasing in $x$, and its associated payoff function

$$
V^{k+1}\left(x \wedge y_{m_{k}}, x \mid \bar{y}_{m_{k}}\right)=\max _{z} V^{k+1}\left(z, x \mid \bar{y}_{m_{k}}\right)
$$

for all the subsequent rounds $k+1 \leq K$. Consequently, if the bidder loses the $k$ th round and observes $\bar{Y}_{m_{k}}=\bar{y}_{m_{k}}$, sequential rationality calls on him to bid $x \wedge y_{m_{k}}$ in round $k+1$ and so on.

We focus on the case $x \leq y_{m_{k-1}}$. (The case with $x>y_{m_{k-1}}$ will result in the bidder bidding $y_{m_{k-1}}$ and win the $k$ th round with certainty.) Suppose the type- $x$ bidder 1 is active in the $k$ th round and bid as though his type was $z\left(\leq y_{m_{k-1}}\right)$. Then his expected payoff is given by $(23)$. Differentiating $V^{k}\left(z, x \mid \bar{y}_{m_{k-1}}\right)$ w.r.t. $z$ yields

$$
\begin{aligned}
& V_{1}^{k}\left(z, x \mid \bar{y}_{m_{k-1}}\right) \\
= & {\left[u^{k}\left(x, z, \bar{y}_{m_{k-1}}\right)-E\left[V^{k+1}\left(x \wedge z, x \mid \bar{Y}_{m_{k}}\right) \mid Y_{m_{k}}=z, \bar{Y}_{m_{k-1}}=\bar{y}_{m_{k-1}}\right]\right] f_{m_{k}}\left(z \mid \bar{y}_{m_{k-1}}\right) } \\
& -\frac{\partial}{\partial z} \varphi^{k}\left(z, \bar{y}_{m_{k-1}}\right)
\end{aligned}
$$

Notice first from (23) that if bidder 1 lost in round $k$ and bid as though his type were equal to the realized $Y_{m_{k}}=z$ in the $(k+1)$ st round, he will win with probability 
1. In this case his expected payoff from the $(k+1)$ st round equals

$$
\begin{aligned}
& E\left[V^{k+1}\left(z, x \mid \bar{Y}_{m_{k}}\right) \mid Y_{m_{k}}=z, \bar{Y}_{m_{k-1}}=\bar{y}_{m_{k-1}}\right] \\
= & \int_{0}^{z} E\left[u^{k+1}\left(x, y, \bar{Y}_{m_{k}}\right) \mid Y_{m_{k}}=z\right] d F_{k+1}(y \mid z)-E\left[\varphi^{k+1}\left(z, \bar{Y}_{m_{k}}, \bar{y}_{m_{k-1}}\right) \mid Y_{m_{k}}=\text { (2] } 8\right)
\end{aligned}
$$

By (4), the first term on the right-hand side of (28)

$$
\int_{0}^{z} E\left[u^{k+1}\left(x, y, \bar{Y}_{m_{k}}\right) \mid Y_{m_{k}}=z\right] d F_{k+1}(y \mid z)=u^{k}\left(x, z, \bar{y}_{m_{k-1}}\right)
$$

Therefore, 27)-(28) imply that the necessary condition for bidding truthfully, $V_{1}^{k}\left(z, z \mid \bar{y}_{m_{k-1}}\right)=$ 0 , is

$$
\frac{\partial}{\partial z} \varphi^{k}\left(z, \bar{y}_{m_{k-1}}\right)=E\left[\varphi^{k+1}\left(z, \bar{Y}_{m_{k}}, \bar{y}_{m_{k-1}}\right) \mid Y_{m_{k}}=z\right] f_{m_{k}}\left(z \mid \bar{y}_{m_{k-1}}\right)
$$

Now we argue that this condition is also sufficient. If bidder 1 bids $z \geq x$, then

$$
\begin{aligned}
& E\left[V^{k+1}\left(x \wedge z, x \mid \bar{Y}_{m_{k}}\right) \mid Y_{m_{k}}=z, \bar{Y}_{m_{k-1}}=\bar{y}_{m_{k-1}}\right] \\
= & E\left[V^{k+1}\left(x, x \mid \bar{Y}_{m_{k}}\right) \mid Y_{m_{k}}=z, \bar{Y}_{m_{k-1}}=\bar{y}_{m_{k-1}}\right] \\
\geq & E\left[V^{k+1}\left(z, x \mid \bar{Y}_{m_{k}}\right) \mid Y_{m_{k}}=z, \bar{Y}_{m_{k-1}}=\bar{y}_{m_{k-1}}\right] \text { by }(26)
\end{aligned}
$$

But then by the assumption that other bidders bid truthfully, (28)-(30) imply

$$
\begin{aligned}
& V_{1}^{k}\left(z, x \mid \bar{y}_{m_{k-1}}\right) \\
\leq & E\left[\varphi^{k+1}\left(z, \bar{Y}_{m_{k}}, \bar{y}_{m_{k-1}}\right) \mid Y_{m_{k}}=z\right] f_{m_{k}}\left(z \mid \bar{y}_{m_{k-1}}\right)-\frac{\partial}{\partial z} \varphi^{k}\left(z, \bar{y}_{m_{k-1}}\right)=0 \text { for } z>x
\end{aligned}
$$

Hence, the bidder has no incentive to bid higher than $x$. Now for $z \leq x$,

$$
\begin{aligned}
& E\left[V^{k+1}\left(x \wedge z, x \mid \bar{Y}_{m_{k}}\right) \mid Y_{m_{k}}=z, \bar{Y}_{m_{k-1}}=\bar{y}_{m_{k-1}}\right] \\
= & E\left[V^{k+1}\left(z, x \mid \bar{Y}_{m_{k}}\right) \mid Y_{m_{k}}=z, \bar{Y}_{m_{k-1}}=\bar{y}_{m_{k-1}}\right]
\end{aligned}
$$

and thus by $(30) V_{1}^{k}\left(z, x \mid \bar{y}_{m_{k-1}}\right)=0$ for all $z \leq x$. So, again, the bidder has no incentive to deviate from bidding $x$. Consequently, integrating (30) yields the characterization of SIC payment rules (9). Note that our induction hypotheses and (30) imply that $\varphi^{k}\left(x, \bar{y}_{m_{k-1}}\right)$ is positive and increasing in $x$.

Finally, we argue that $\varphi^{k}$ is unique under any SIC payment rules $p^{k}$ such that $p^{k}(0, \cdot) \equiv 0$. For $k=K$, given any $\bar{y}_{m_{K-1}}$ the right-hand side of 25$)$ determines 
a unique function $\varphi^{K}\left(\cdot, \bar{y}_{m_{K-1}}\right)$. Thus, all sequential auctions must have the same round- $K$ expected revenue. By induction, we see easily from (9) that the values of all $\varphi^{k}\left(x, \bar{y}_{m_{K-1}}\right)$ are uniquely determined. Consequently, given the number of rounds $K$ and the number of $\ell_{k}$ objects to be sold in round $k=1, \ldots, K$, all SIC payment rules $p^{k}$ such that $p^{k}(0, \cdot) \equiv 0$ induce the same $k$ th round expected payment.

\section{Appendix B. Revenue Equivalence}

In this appendix, we discuss in more detail of the revenue equivalence result of Corollary 1, and provide an instructive proof of the second part of this corollary concerning the irrelevance of the sequencing rules. In this corollary, we report two revenue equivalence results. The first one is a straightforward extension of the payoff equivalence theorem for single-round auctions, which holds that given any sequencing rule and updated information, each active bidder's expected payment in a given round of the sequential auction is the same under any payment rule for this round $4^{15}$ Consequently, as long as the sequencing rule is fixed, all SIC payment rules imply the same expected revenue from each round ${ }^{16}$ The second prediction of Corollary 1 holds that the total expected revenue is the same under any sequencing rules. In particular, revenue equivalence holds between any sequential auction and the simultaneous multi-unit auction. ${ }^{17}$ In light of the fact that the expected payment/revenue can, predictably, differ across different rounds at a sequential auction, it is of interest to understand how and why sequencing tactics do not give the seller a chance to extract more rents from the bidders. We provide a (novel) proof of Corollary 1 here that helps make

\footnotetext{
${ }^{15}$ E.g., Vickrey (1961), Myerson (1981), Riley and Samuelson (1981) for single-unit auctions, and Krishna and Maenner (2001) for single-period incentive compatible mechanisms, among many others.

${ }^{16}$ This result extends, e.g., Krishna (2010, Chapter 15) for the case with independent private values, a single-unit sale per round, and first-price vs. second-price payment rules.

${ }^{17}$ Similar conclusions have been shown for the private values cases (e.g., Weber, 1983; Maskin and Riley, 1989; Bulow and Klemperer, 1994). The fact that the sequencing rules do not matter in these cases can also be seen as a consequence of the martingale property of the price sequences.
} 
out the logic behind the result: all sequencing and payment rules must satisfy the SIC constraints, under which every bidder effectively faces the same probability of winning an object prior to the auction. Consequently, since the lowest-type bidder pays and receives nothing, the total expected revenue must be invariant to sequencing and payment rules by the revenue equivalence theorem. ${ }^{18}$

Proof of Corollary 1. We focus on part (ii), since part (i) is obvious. In this alternative proof, we compare explicitly the expected revenues from two auction policies, one with $K=1$ so that it is a simultaneous auction of all $m$ objects, the other with an arbitrary sequencing rule $K>1$ selling $\ell_{k}$ objects in round $k=1, \ldots, K$. Let $\Phi_{1}(x)$ and $\Phi_{K}(x)$ denote the total pre-auction expected payment, respectively, at these auctions by bidder 1 with type $x$. We prove a stronger result of payoff equivalence that $\Phi_{1}(x) \equiv \Phi_{K}(x)$, which implies the irrelevance of sequencing rules to the seller's total expected revenue.

Under simultaneous sales, the condition (10) for $K=1$ reduces to:

$$
\Phi_{1}(x)=E\left[v\left(Y_{m}, \bar{Y}\right) \mid\left\{Y_{m}<x \leq 1\right\}\right]
$$

Under sequential sales, (10) implies

$$
\varphi^{k}\left(x, \bar{y}_{m_{k-1}}\right)=E\left[v\left(Y_{m}, \bar{Y}\right) \mid \bar{Y}_{m_{k-1}}=\bar{y}_{m_{k-1}},\left\{Y_{m_{k}}<x \leq y_{m_{k-1}}\right\}\right]
$$

At the start of the auction, for bidder 1 with type $x$, his round- $k$ expected payment is a random variable $\tilde{\varphi}^{k}(x)$. It is a function of $\bar{Y}_{m_{k-1}}$ such that

$$
\tilde{\varphi}^{k}(x)= \begin{cases}\varphi^{k}\left(x, \bar{Y}_{m_{k-1}}\right) & \text { if } x \leq Y_{m_{k-1}} \\ 0 & \text { if } x>Y_{m_{k-1}}\end{cases}
$$

Thus, prior to the auction, a bidder with type $x$ has an expected payment in the $k$ th

\footnotetext{
${ }^{18}$ Mezzetti (2011, Lemma 1) uses this logic to establish payoff equivalence in sequential auctions under interdependent values (the case of PIE), where bidders are averse to price risks. Our result extends readily to Mezzetti's case with generalized interdependent values.
} 
round equal to

$$
\begin{aligned}
& E\left[\tilde{\varphi}^{k}(x)\right] \\
= & E\left[\varphi^{k}\left(x, \bar{Y}_{m_{k-1}}\right) \mid\left\{x \leq Y_{m_{k-1}}\right\}\right] \\
= & E\left[E\left[v\left(Y_{m}, \bar{Y}\right) \mid \bar{Y}_{m_{k-1}}=\bar{y}_{m_{k-1}},\left\{Y_{m_{k}}<x \leq y_{m_{k-1}}\right\}\right] \mid\left\{x \leq Y_{m_{k-1}}\right\}\right] \text { by } 32 \\
= & E\left[v\left(Y_{m}, \bar{Y}\right) \mid\left\{Y_{m_{k}}<x \leq Y_{m_{k-1}}\right\}\right]
\end{aligned}
$$

The sum of the expected payments in all rounds gives the bidder's total pre-auction expected payment:

$$
\Phi_{K}(x)=\sum_{k=1}^{K} E\left[v\left(Y_{m}, \bar{Y}\right) \mid\left\{Y_{m_{k}}<x \leq Y_{m_{k-1}}\right\}\right]
$$

Since the event $\left\{Y_{m}<x \leq 1\right\}$ can be partitioned into $K$ sub-events:

$$
\left\{Y_{m}<x \leq 1\right\}=\bigcup_{k=1}^{K}\left\{Y_{m_{k}}<x \leq Y_{m_{k-1}}\right\} \quad\left(Y_{m_{0}}=1, Y_{m_{K}}=Y_{m}\right)
$$

we derive from $(31)$ and $(33)$ that

$$
\begin{aligned}
\Phi_{1}(x) & =E\left[v\left(Y_{m}, \bar{Y}\right) \mid\left\{Y_{m}<x \leq 1\right\}\right] \\
& =\sum_{k=1}^{K} E\left[v\left(Y_{m}, \bar{Y}\right) \mid\left\{Y_{m_{k}}<x \leq Y_{m_{k-1}}\right\}\right]=\Phi_{K}(x)
\end{aligned}
$$

The seller's total expected revenue is therefore the same from either auction.

\section{References}

Ashenfelter, Orley. 1989. "How Auctions Work for Wine and Art." Journal of Economic Perspectives, 3: 23-36.

Ashenfelter, Orley, and David Genesove. 1992. "Testing for Price Anomalies in Real-Estate Auctions." American Economic Review, 82: 501-505.

Athey, Susan. 2001. "Single Crossing Properties and the Existence of Pure Strategy Equilibria in Games of Incomplete Information." Econometrica, 69: 861-889. 
Beggs, Alan, and Kathryn Graddy. 1997. "Declining Values and the Afternoon Effect: Evidence from Art Auctions." Rand Journal of Economics, 28: 544-565.

Bernhardt, Dan, and David Scoones. 1994. "A Note on Sequential Auctions." American Economic Review, 84: 501-505.

Black, Jane, and David de Meza. 1992. "Systematic Price Differences Between Successive Auctions Are No Anomaly." Journal of Economics and Management Strategy, 1: 607-28.

Bulow, Jeremy, and Paul Klemperer. 1994. "Rational Frenzies and Crashes." Journal of Political Economy, 102(1): 1-23.

Chanel, Oliver, Louis-Andre Gérard-Varet, and Stephanie Vincent. 1996. "Auction Theory and Practice: Evidence from the Market for Jewelry," in Economics of the Arts: Selected Essays. Victor Ginsburgh and Pierre-Michel Menger, eds. Amsterdam: Elsevier, pp. 135-49.

Deltas, George, and Georgia Kosmopoulou. 2004. “Catalogue' vs 'Order-of-sale' effects in Sequential Auctions: Theory and Evidence from a Rare Book Sale. Economic Journal, 114: 28-54.

DeMarzo, Peter M., Ilan Kremer, and Andrzej Skrzypacz. 2005. "Bidding with Securities: Auctions and Security Design." American Economic Review, 95: 936-959.

Engelbrecht-Wiggans, Richard. 1994. "Sequential Auctions of Stochastically Equivalent Objects." Economics Letters, 44: 87-90.

Engelbrecht-Wiggans, Richard, and Charles M. Kahn. 1999. "Calibration of a Model of Declining Prices in Cattle Auctions." Quarterly Review of Economics and Finance, 39: 113-128. 
Eso, Peter, and Lucy White. 2004. "Precautionary Bidding in Auctions." Econometrica, 72: 77-92.

Gale, Ian L., and Donald B. Hausch. 1994. "Bottom-Fishing and Declining Prices in Sequential Auctions." Games and Economic Behavior, 7: 318-331.

Gale, Ian L, and Mark Stegeman. 2001. "Sequential Auctions of Endogenously Valued Objects." Games and Economic Behavior, 36: 74-103.

Gandal, Neil. 1997. "Sequential Auctions of Interdependent Objects: Israeli Cable Television Licences." Journal of Industrial Economics, 45: 227-44.

Ginsburgh, Victor A. 1998. "Absentee Bidders and the Declining Price Anomaly in Wine Auctions." Journal of Political Economy, 106: 1302-1319.

Goeree, Jacob K. 2003. "Bidding for the Future: Signaling in Auctions with an Aftermarket." Journal of Economic Theory 108: 345-364.

Holt, Charles A. 1980. "Competitive Bidding for Contracts under Alternative Auction Procedures." The Journal of Political Economy, 88: 433-445.

Hu, Audrey, and Liang Zou. 2015. "Sequential Auctions, Price Trends, and Risk Preferences." Journal of Economic Theory 158: 319-335.

Hu, Audrey, Steven A. Matthews, and Liang Zou. 2015. "English Auctions with Ensuing Risks and Heterogeneous Bidders." University of Pennsylvania PIER Working Paper 15-010.

Hu, Audrey, Theo Offerman, and Liang Zou. 2014. "How Risk Sharing May Enhance Efficiency in English Auctions." Tinbergen Institute Discussion Paper, TI 2014015/I.

Jeitschko, Thomas D., and Elmar Wolfstetter. 1998. "Scale Economies and the Dynamics of Recurring Auctions." Economic Inquiry, 40: 403-14. 
Jones, Chris, Flavio M Menezes, and Francis Vella. 2004. "Auction Price Anomalies: Evidence from Wool Auctions in Australia." Economic Record, 80: 271-288.

Krishna, Vijay. 2003. "Asymmetric English Auctions." Journal of Economic Theory, 112: $261-288$.

Krishna, Vijay. 2010. Auction Theory. Second Edition, Elsevier: Academic Press.

Krishna, Vijay, and Eliot Maenner. 2001. "Convex Potentials with an Application to Mechanism Design." Econometrica 69: 1113-1119.

Kittsteiner, Thomas, Joerg Nikutta, and Eyal Winter. 2004. "Declining Valuations in Sequential Auctions." International Journal of Game Theory, 33: 89-106.

Maskin, Eric, and John Riley. 1989. "Optimal Multi-Unit Auctions.” In Frank Hahn (ed.), The Economics of Missing Markets, Information, and Games. Oxford: Oxford University Press, Clarendon Press.

Menezes, Flavio M. and Monteiro, Paulo K. 2003. "Synergies and Price Trends in Sequential Auctions." Review of Economic Design, 8: 85-98.

Mezzetti, Claudio. 2011. "Sequential Auctions with Informational Externalities and Aversion to Price Risk: Decreasing and Increasing Price Sequences." Economic Journal, 121: 990-1016.

Mezzetti, Claudio, Aleksandar Saša Pekeč, and Ilia Tsetlin. 2008. "Sequential vs Single-Round Uniform-Price Auctions." Games and Economic Behavior, 62: 591-609.

McAfee, R. Preston, and Daniel Vincent. 1993. "The Declining Price Anomaly." Journal of Economic Theory, 60: 191-212.

McAfee, R. Preston, and Daniel Vincent. 1997. "Sequentially Optimal Auctions" Games and Economic Behavior, 18: 246-276. 
Milgrom, Paul. 2004. Putting Auction Theory to Work. Cambridge: Cambridge University Press.

Milgrom, Paul, and Robert J. Weber. 1982. "A Theory of Auctions and Competitive Bidding." Econometrica, 50: 1089-1122.

Milgrom, Paul, and Robert J. Weber. 2000. "A Theory of Auctions and Competitive Bidding II," in The Economic Theory of Auctions Vol. II. Klemperer Paul. ed. Edward Elgar Publishing Ltd, pp. 179-194.

Milgrom, Paul, and Chris Shannon. 1994. "Monotone Comparative Statics." Econometrica, 62: 157-180.

Myerson, Roger B. 1981. "Optimal Auction Design." Mathematics of Operations Research 6: 58-73.

Pavan, Alessandro, Ilya Segal, and Juuso Toikka. 2014. "Dynamic Mechanism Design: A Myersonian Approach." Econometrica, 82: 601-653.

Pitchik, Carolyn, and Andrew Schotter. 1988. "Perfect Equilibria in BudgetConstrained Sequential Auctions: An Experimental Study." RAND Journal of Economics, 19: 363-388.

Rosato, Antonio. 2014. "Loss Aversion and the 'Afternoon Effect' in Sequential Auctions." University of Technology Sydney, Working Paper.

van den Berg, Gerard. J., Jan C. van Ours, and Menno P. Pradhan. 2001. "The Declining Price Anomaly in Dutch Dutch Rose Auctions." American Economic Review, 91: 1055-62.

Weber, Robert J. 1983. "Multi-Object Auctions." in Auctions, Bidding and Contracting: Uses and Theory. Richard Engelbrecht-Wiggans, Martin Shubik, and Robert M. Stark, eds. New York: New York University Press, pp. 165-194. 